\title{
Las cuentas de la Biblioteca del Real Colegio de Cirugía de la Armada de Cádiz (1748-1844). Una breve reseña
}

\author{
Ana Remón Rodríguez \\ Jefa de Sección de Colección y Patrimonio de la Biblioteca de la Universidad de \\ Cádiz \\ ana.remon@uca.es
}

RESUMEN: Se estudia los ingresos y gastos de la Biblioteca del Real Colegio de Cirugía de Cádiz hasta la creación de la Facultad de Ciencias Médicas (1748-1844). La reconstrucción de la gestión de las cuentas de la Biblioteca se ha realizado a través del análisis de los documentos manuscritos de la época, tanto de los libros de actas como los de cuentas.

Palabras clave: Colegio de Cirugía de Cádiz (1748-1844), Biblioteca Colegio de Cirugía de Cádiz (1748-1844), Bibliotecas Médicas Siglo XVIII, Contabilidad.

\section{The accounts of the Library of the Royal College of Surgery of the Armada de Cádiz (1748-1844). A brief review}

ABSTRACT: The account of the Library of the Royal College of Surgery of Cádiz is studied, until the creation of the Faculty of Medical Sciences (1748-1844). The reconstruction of the management of the Library has been carried out through the study of the manuscript documents of the time, both of the minute and account books.

Keywords: College of Surgery of Cadiz (1748-1844), Library College of Surgery of Cadiz (1748-1844), Medical Library eighteenth century, Accounting.

\section{INTRODUCCIÓN}

El Real Colegio de Cirugía de la Armada de Cádiz, es una institución ilustrada perteneciente a la Armada Española. Puso las bases para que la Cirugía Española pasara de ser una actividad práctica y artesanal a una profesión equiparable a la Medicina. 
Siendo una institución militar, que actúa en el ámbito civil a nivel nacional, tuvo a su vez una proyección internacional a través de la labor que llevaron a cabo sus profesores.

Nuestro trabajo se centra en la Biblioteca, la cual juega un papel fundamental para llevar a cabo el objetivo propuesto por Juan Lacomba ${ }^{1}(1680-1748)$ y Pedro Virgili (1699-1776). La propuesta de ambos es salir de la situación decadente en la que se encuentra la práctica quirúrgica en España y elevar la cirugía y los cirujanos a un nivel europeo. Basado en un sistema de formación estricto y con una metodología concreta, prestigiando la profesión a través de una formación adecuada, donde fuera posible la adquisición de nuevos saberes a través de los conocimientos de otros profesionales. Utilizando las fuentes de información más avanzadas de la época, los libros.

La Biblioteca juega un papel importante, pues es un elemento crucial para consolidar la formación teórica que se trasmite a través de los documentos escritos. Los libros son la base teórica y un complemento de la práctica profesional.

En esta breve aportación hablaremos del aspecto económico tan necesario para hacer posible la creación de una biblioteca. La cual hemos podido reconstruir a través del estudio de los documentos manuscritos de la época, tanto los libros de Actas como los de Cuentas, así como los primeros inventarios localizados, de donde hemos extraídos el principal núcleo de la información de la colección fundacional. El período de estudio ocupa toda la existencia del Colegio de Cirugía que acaba como Facultad de Ciencias Médicas, con una duración aproximada de un siglo, entre los años 1748 y 1844.

\section{LOS INICIOS DE LA COLECCIÓN DE LA BIBLIOTECA}

A través de los Libros de cuentas y de caudales del Colegio ${ }^{2}$, hemos podido conocer la economía referida a la parte de la Biblioteca. Desde un principio Virgili comprende que para crear una Biblioteca es necesario un presupuesto específico y que se mantenga en el tiempo. Su idea de formar pronto una colección básica para funcionar como biblioteca no sería fácil contando solo con el dinero procedente de la Real Hacienda destinado al sostén del Colegio. Así propone en 1749 que cada miembro del personal del Colegio aporte una cantidad destinada a formar la colección de libros de la Biblioteca y la de los instrumentos. En cierto modo introduce la idea de un mecenazgo a nivel individual para contribuir a una causa noble que repercutirá en el bien de todos, pues la Real Hacienda no puede con todo y es necesario una ayuda para poner en marcha los planes que tiene para el Colegio.

\footnotetext{
${ }^{1}$ Jean Lacombe, nombre que se ha castellanizado a Juan Lacomba en los diferentes escritos.

${ }^{2}$ Existen 5 libros de cuentas, 4 de caudales y 1 de gestión de los intereses del fondo que cubren la economía del colegio desde los años 1751 a 1844. Fuente: Archivo de la Universidad de Cádiz (AUCA). Disponible: https://fondo-antiguo.uca.es/flora/jsp/index_fondo_antiguo.jsp 
Esta aportación económica será diferente dependiendo del estatus del personal dentro de la institución (Imagen 1), cada uno debe aportar ${ }^{3}$ un 1\% de su sueldo: para el ayudante primero 10 pesos, para el segundo 5 pesos, los colegiales 2,5 pesos y 25 pesos los que se embarcasen, los cuales lo entregarían a la vuelta de la travesía, esto dará como resultado que no siempre los ingresos tengan una cantidad fija anual, dependerá de los períodos que tengan las travesías, y así queda reflejado en ellos.

El acuerdo es aceptado y firmado el 27 de enero de $1751^{4}$, por el Cirujano Mayor, el Director del Colegio, maestros ayudantes primero y segundo de la Armada. Virgili solicita autorización sobre este asunto al Marqués de la Ensenada, y éste en una carta de contestación le indica que al tratarse de un acuerdo interno no tiene que ser aprobado por el Rey, al no denegarlo lo apoya ${ }^{5}$, por lo que se pondrá en marcha ese mismo año.

Para la recogida de este dinero, se acuerda que exista una caja de caudales donde se deposite la cantidad con la que los profesores y colegiales contribuyan con el $1 \%$ de su sueldo para la compra de libros y máquinas. Dicha caja tendrá tres llaves, una para el Director del Colegio, la segunda para el Intendente y la tercera para el Bibliotecario, este último intervendrá en toda la gestión de este fondo, pues es el encargado de las compras de los libros, así como de su impresión y venta, que se gestionan desde la Biblioteca. El Bibliotecario ${ }^{6}$ por tanto aparecerá en todos los resúmenes de cuentas que se realizan anualmente. A la Biblioteca se le asignará de forma fija un $3 \%$ de ese fondo anualmente.

Este acuerdo comenzó en 1751 y se mantienen a lo largo de los años (Imagen 1), pues se hizo por costumbre, y los nuevos que se fueron incorporando lo aceptaron. Pasado el tiempo se oyeron voces de que también ese caudal se podía utilizar para otros asuntos necesarios, como el socorro a las viudas de los cirujanos en los casos que fuera necesario, y parece que esto hizo que ya no existiera tanta voluntariedad, y que algunos dejaran de pagar.

Ante esta situación Virgili realiza un escrito al Rey en diciembre de 1764, en el que explica la situación expuesta anteriormente. En marzo de 1765, hay una contestación del Rey, en la que Virgili sale favorecido, pues el Rey declara que dicho acuerdo es inalterable. Aunque en un principio se hizo de forma voluntaria, la repetición del acto lo convierte en obligatorio. Con lo que se consigue, que la voluntariedad, se convierta en obligación (Márquez Espinós y Orozco Acuaviva 1983).

Las ordenanzas de 1791 (Real Colegio de Medicina y Cirugía de Cádiz, 1791), reconocen un presupuesto para la compra de libros realizada del fondo del caudal del Colegio, independiente del que proviene de la Real Hacienda, y así lo veremos reflejado en los inventarios y libros de cuentas del Colegio. Existe un estado de cuentas

\footnotetext{
${ }^{3}$ Archivo de la Universidad de Cádiz (AUCA). Libro [copiador] de Reales Órdenes y decretos en favor del Real Hospital Cirugía, Real Colegio Seminario de Cádiz e individuos que lo componen: 17081763. L-54 RC, 1749, p. 126.

${ }^{4}$ Ibidem, 1751, p. 149.

${ }^{5}$ Ibidem.1751, p. 150.

${ }^{6}$ Archivo de la Universidad de Cádiz (AUCA). Libro de noticias de propuestas y deliberaciones, así como de los gastos del Real Colegio de Cirugía: 1751-1814. [Libro primero]. L-22 RC, pp. 10-11. 
gestionado por el Bibliotecario ${ }^{7}$, donde se llevan los ingresos y los gastos producidos del fondo del caudal correspondiente al 1\%, relacionados con la Biblioteca.

\section{Imagen 1. Convenio propuesto por Virgili para aportar fondos al Colegio}

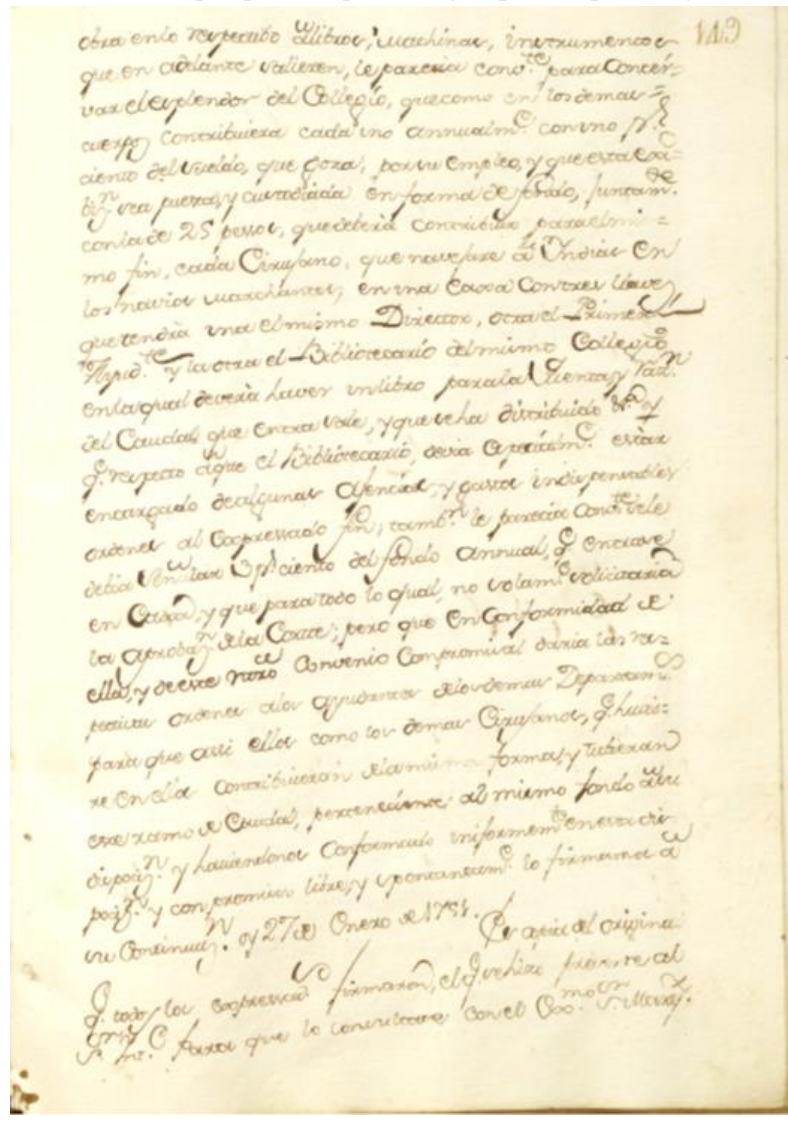

Fuente: Archivo de la Universidad de Cádiz (AUCA). Reales Órdenes y decretos, L-54 RC, p. 126.

\section{LAS ENTRADAS Y SALIDAS EN LOS LIBROS DE CUENTAS}

A través de los Libros de cuentas, hemos podido obtener los distintos conceptos para las entradas y las salidas, que son las siguientes:

Entradas:

- Las aportaciones del $1 \%$ del personal.

- Las ventas de libros a nivel interno del colegio, que se realizan desde la Biblioteca ${ }^{8}$.

- Las ventas exteriores con otros colegios como el de Madrid, Barcelona y América.

- La aportación para la Biblioteca del presupuesto de la Real Hacienda.

\section{Salidas:}

${ }^{7}$ Archivo de la Universidad de Cádiz (AUCA). Libro de noticias de propuestas y deliberaciones, así como de los gastos del Real Colegio de Cirugía. Año: 1751-1814 [Libro 1]. L-22 RC, pp. 10-11.

8 Archivo de la Universidad de Cádiz (AUCA). Libro de cuentas del real Colegio de Cirugía. Años 1792-1836. [Libro 35], 1772. L-46 RC, p. 443.

DOI del artículo:
$\underline{\text { http://dx.doi.org/10.25267/Cuad investig fondos arch UCA.2 }}$
$\underline{019 . i 1.05}$


- La adquisición de obras nuevas, que es la que se lleva el mayor presupuesto.

- Compra de instrumental que también era costeado de este fondo. Traído principalmente del extranjero, pero no gestionado directamente por el Bibliotecario.

- Plantas y herbarios, estos últimos formaron parte de la colección de la Biblioteca, las plantas iban destinadas al Jardín Botánico del Colegio.

- Impresión de manuales para los alumnos, elaborados por los profesores del Colegio. La venta de los libros tiene dos procedimientos: uno referido a las obras que se imprimen supervisadas por el Colegio y que costea en su totalidad; y otro el que directamente gestiona e imprime cada profesor de forma particular. De este último solo se beneficia el Colegio en una parte del producto de su venta.

- Impresiones menores 9 : oraciones fúnebres e inaugurales; sorteo de materias; programas; papeletas; anuncios de conferencias; papel de marca para los oficios del Colegio, etc.

- Encuadernaciones ${ }^{10}$ de las obras adquiridas, y de las que imprime el propio Colegio.

- Gastos menores de administración y gestión, que incluyen: oficios, gastos de aduanas, pagos al Tribunal del Santo Oficio, portes de libros en los diversos traslados, etc.

- Compra de enseres necesarios para la mejora de la instalación bibliotecaria.

- Aportación al Bibliotecario, para la que en un principio Virgili propone la cantidad de 50 escudos $^{11}$. Dicha cantidad se modifica a lo largo del tiempo. En las Ordenanzas de 1764 se indica que será de 2.000 reales de vellón, y para su ayudante 1.000 .

El sostén mayoritario de la Biblioteca proviene de las aportaciones del personal. El convenio realizado fue la principal fuente de ingresos del Real Colegio (Ferrer 1983).

\section{DE LA PROSPERIDAD A LA DECADENCIA}

Existen los estados de cuentas del fondo desde los años 1751 hasta 1844 (Gráfico 1), (Ferrer 1867b). Hemos constatado consultando el Archivo de la Universidad de Cádiz que se encuentran registrados en su catálogo: cinco libros de cuentas, cuatro de caudales y uno de gestión de los intereses del fondo que cubren la contabilidad del Colegio desde los años 1751 a $1844^{12}$. Los gastos y los ingresos son descritos anualmente en los libros de cuentas. La información de los gastos varía en cuanto al detalle de la descripción dependiendo del año. En algunos se consignan datos de las obras como título y precio

${ }^{9}$ Ibidem, 1771, p. 405.

${ }^{10}$ Ibidem, 1773, p. 507.

${ }^{11}$ Archivo de la Universidad de Cádiz (AUCA). Libro [copiador] de Reales Órdenes y decretos en favor del Real Hospital Cirugía, Real Colegio Seminario de Cádiz e individuos que lo componen: 17081763. L-54 RC, 1749, p. 125.

${ }^{12}$ Archivo de la Universidad de Cádiz (AUCA). Relación de documentos referidos al Real Colegio de Cirugía. Disponible: https://fondo-antiguo.uca.es/flora/jsp/index_fondo_antiguo.jsp 
(imagen 3) $)^{13}$, y en otros años se dice que se han comprado obras para la Biblioteca, con descripciones muy someras, en las no se puede saber ni la cantidad de obras adquiridas, ni procedencia, ni descripción bibliográfica de las obras.

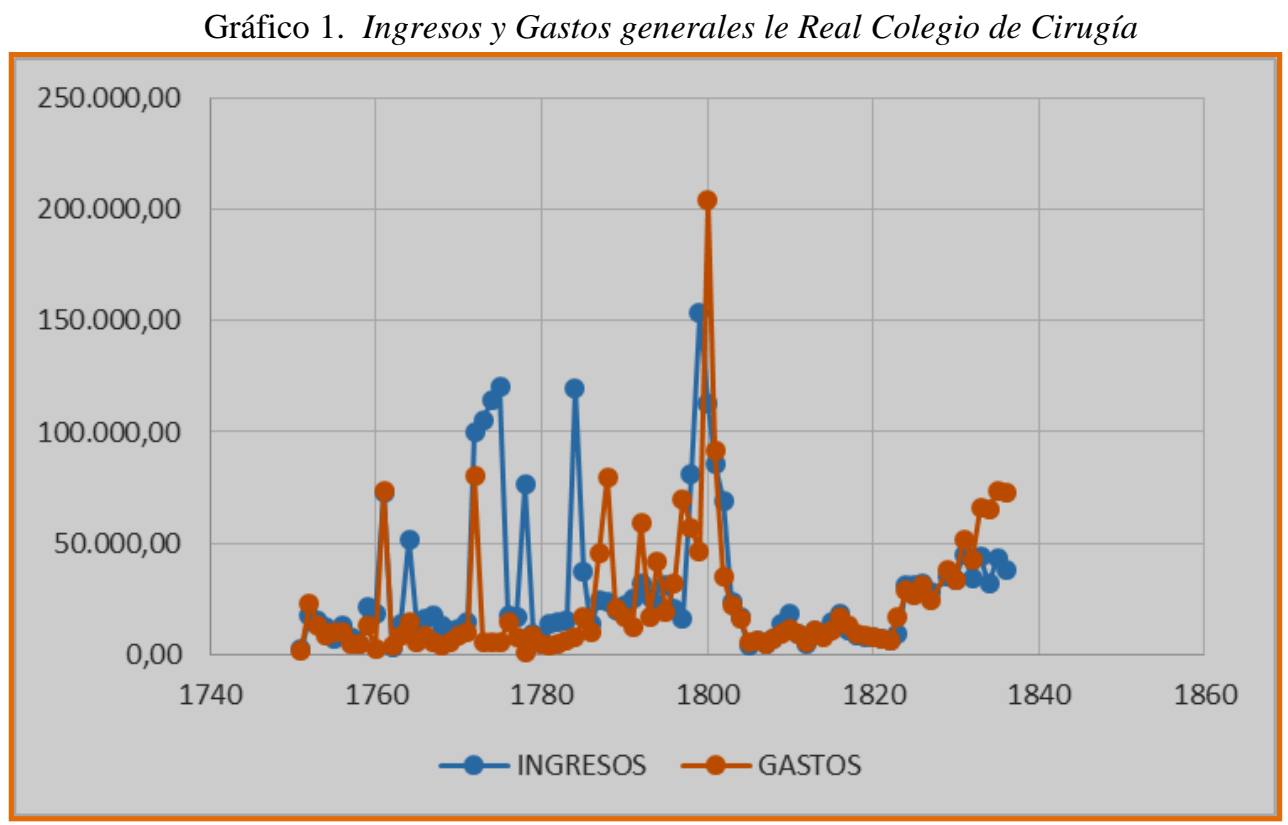

Fuente: Archivo de la Universidad de Cádiz. (AUCA) Libros de Cuentas. Elaboración propia, 2015

El movimiento económico de la Biblioteca (Gráfico 1), refleja que la actividad se inicia con gran diligencia, y es en los primeros momentos de su creación cuando se produce un número mayor de entrada de obras. En los inicios las entradas y los gastos están muy ajustados, se producen débitos en el pago de las obras, que se abonan posteriormente, es importante tener en cuenta que las obras se solicitan, pero pueden tardar en llegar hasta un año, acumulándose el gasto para el año siguiente o posterior.

Aproximadamente a los 20 años de creación de la Biblioteca se produce un descenso en las compras (Gráfico 1), que coincide con una gran producción de manuales por parte del Colegio, esto quiere decir que el presupuesto se destina a la edición de sus propias obras. Al final del siglo habrá otro remonte de nuevas adquisiciones, hacía el año 1795. Es necesario resaltar que a lo largo de todo el período no cesan las entradas de documentos en mayor o menor cantidad.

Entendemos que la compra de las nuevas obras está directamente relacionada con la necesidad de formación y puesta al día de los profesores, que las tenían que utilizar como obras recomendadas para su asignatura (Real Colegio de Medicina y Cirugía de Cádiz, 1791).

La crisis económica reflejada en el presupuesto de la Biblioteca es la propia crisis del Colegio, algunas debidas a causas externas, como son las epidemias de principio de

13 Archivo de la Universidad de Cádiz (AUCA). Libro de Cuentas del Real Colegio de Cirugía de Cádiz. 1751-1780. [libro 32].L-44 RC, p.11 
siglo; la Guerra de la Independencia; la decadencia económica de la Armada, que no puede hacerse cargo de la magna organización que ha formado; la inestable gobernabilidad de Fernando VII; y los cambios en la gestión del Colegio al depender de otras instituciones del estado. También hubo causas internas como los cambios que sufre la institución debido a vaivenes en los planes de estudio, y a las luchas continuas con el protomedicato y la competencia con los otros colegios que se habían ido creando (Orozco Acuaviva 2001).

La situación inestable se empieza a notar a partir del año 1803 (Gráfico 1), y se extiende hasta el cierre del Colegio. Desde 1833, la situación económica se hace más crítica, se vive una situación de provisionalidad enorme, en estos años aparece muchas veces en las actas, que no hay dinero para la Biblioteca ${ }^{14}$. En este período con el único dinero que se cuenta es con la contribución de los colegiales y con el 3\% de lo que se saca de la venta de los libros, que según las ordenanzas su producto es para el Colegio (Real Colegio de Medicina y Cirugía de Cádiz 1791).

Así llegamos al año $1837^{15}$ en la que se indica el deplorable estado de los fondos del Colegio y la situación económica de enorme penuria por las que atraviesa. Tanto es así que no se pueden comprar dos obras solicitadas al profesor de Botánica.

Las circunstancias de la política económica de España, unida a la creación de la Junta Superior Gubernativa (Campos Díez 1999), que regirá todos los colegios, perjudica al de Cádiz por la centralización del presupuesto. En estos momentos de dificultad económica se emprenden acciones encaminadas a animar a los colegiales a que ayuden a la economía del Colegio y compren los libros que se publican y que vende el mismo: las obras de Canivell, Villaverde, etc.; así como que no se deje de pagar la aportación del $1 \%$ por parte del personal del Colegio. Hasta en momentos de crisis, el personal al ser admitidos en el Colegio, aceptaban la aportación de seis reales de vellón, dicho dinero continuó aplicándose al mismo concepto para el que fue creado: "se aplicará exclusivamente a la adquisición de obras eso se hace indispensable para la ilustración de esta escuela, también se dice que este dinero irá destinado a las obras necesarias en la Biblioteca y para la restauración de los libros deteriorados" ${ }^{\text {"16 }}$. Hasta el final de la institución en el año de 1843 se mantendrá esta contribución, siendo un pilar fundamental en todo momento para el sostenimiento de la Biblioteca.

También se propugna el intercambio de las obras con los otros Colegios de Barcelona y Madrid, para que allí se puedan vender las obras publicadas, y así sacar algo más de dinero. Hay un envío importante en $1834^{17}$ a Barcelona, pues hay un déficit

\footnotetext{
${ }^{14}$ Archivo de la Universidad de Cádiz (AUCA). Libros de Actas de la [Junta] del Real Colegio de Cirugía y Real Colegio de Medicina y Cirugía, años: 1832-1836 [libro Tercero]. L-25RC, p. 132.

${ }^{15}$ Archivo de la Universidad de Cádiz (AUCA). Libro copiador de actas de la Junta Escolástica del Colegio Nacional de Medicina y Cirugía, año 1837-1840, [Libro quinto], año 1837. L-26 RC, p. 26.

${ }^{16}$ Archivo de la Universidad de Cádiz (AUCA). Libro de actas de la [Junta] del Real Colegio de Cirugía y Real Colegio de Medicina y Cirugía. Años 1725-1832[Libro Tercero], año 1831.L-24 RC, p. 204.

${ }^{17}$ Archivo de la Universidad de Cádiz. Libros de Actas de la [Junta] de la Junta del Colegio Nacional de Medicina y Cirugía. Años: 1832-1836. [libro Cuarto].1837 L-26 RC, p. 27.
}

\section{DOI del artículo: \\ http://dx.doi.org/10.25267/Cuad investig fondos arch UCA.2}


y se intentan vender las obras en existencias, ya que la situación es delicada y no se puede realizar ningún gasto.

Sin embargo, Diego Ferrer indica que la decadencia económica del Colegio comienza un poco antes ya en 1796 ,con la puesta en marcha de la Real Junta Superior Gubernativa de los Reales Colegios del Reino con un carácter totalmente centralista, y esto perjudica al Colegio de Cádiz, ya que es el que tiene mayor número de alumnos, y por lo tanto el que aporta más, sin embargo no es el más beneficiado, teniendo que compensar las economías más precarias de otros Colegios (Ferrer 1967a).

La recuperación económica se producirá en el año de $1842^{18}$, cuando de nuevo se aprueba un presupuesto para la Biblioteca y más tarde en el año 1844 se envían al Colegio 1.870 reales de la Junta de Centralización de fondos de Instrucción Pública ${ }^{19}$, ya como Facultad de Ciencias Médicas.

Las aportaciones procedentes de la venta de libros y las cuotas de los miembros del Colegio serán un pilar imprescindible para el sostenimiento económico de la Biblioteca a lo largo de toda su existencia (Imagen 1).

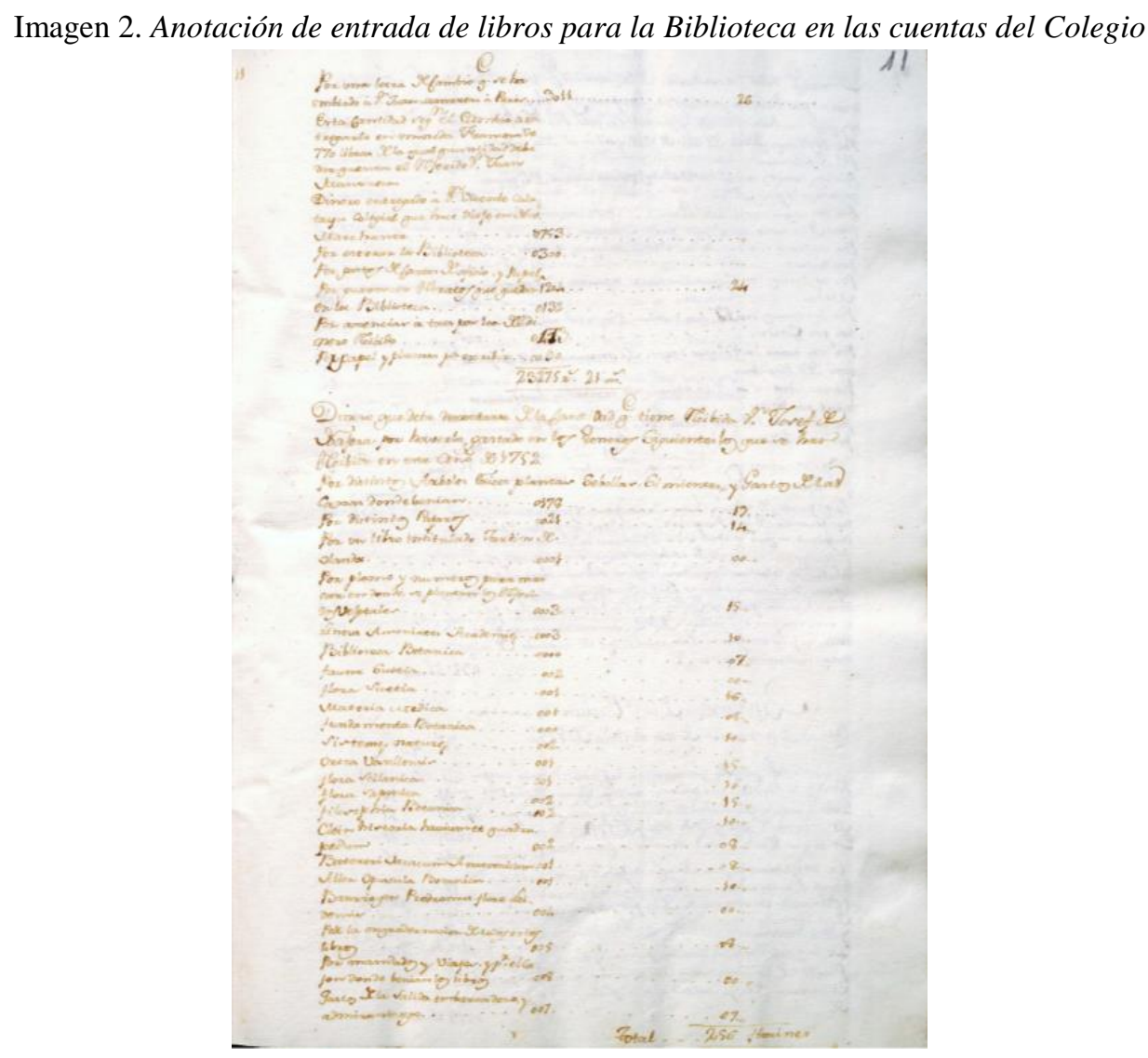

Fuente: Archivo de la Universidad de Cádiz (AUCA). Libro de Cuentas, L-44 RC, p. 11

\footnotetext{
${ }^{18}$ Ibidem, 1842. L-26 RC, p. 116.

${ }^{19}$ Archivo de la Universidad de Cádiz (AUCA). Libro de Actas de la [Junta] del Colegio Nacional de Medicina y Cirugía. Años 1840-1844- [Libro Sexto], 1844. L-27 RC, p. 226.
} 
Desde el mismo momento de su fundación y hasta el final de la institución la vida de la Biblioteca se mantiene activa, sobre todo en sus inicios y hasta final del siglo XVIII, hay que reconocer el impulso dado por Virgili y la continuación de su plan por los profesores que continuaron su obra, esto hace que se prolongue a lo largo de toda su existencia. En el siglo XIX, se inicia un periodo de decadencia que se aprecia en la propia gestión de la Biblioteca (Gráfico 1).

En conjunto podemos decir que la Biblioteca mantuvo una vida muy activa, salvo en momentos de crisis, llegando a formar una colección muy selecta, en continuo crecimiento y adaptada a las necesidades de sus usuarios.

Pudo tener una trayectoria muy parecida a una Biblioteca que se creó en la misma zona y período histórico, amparadas ambas bajo la remodelación General de la Marina iniciadas por Patiño a partir del año 1717, que fue la biblioteca de La Academia de Guardias Marinas de Cádiz (1717-1827). Este se convirtió en un centro de enseñanza especializado y en un foco de difusión de la Ciencia; su biblioteca en 1775, poseía 1.282 volúmenes, donde se encontraban los autores más destacados de la ciencia moderna y los mejores tratados de navegación (González González 2010). En este mismo periodo la Biblioteca del Real Colegio poseía aproximadamente, al final del siglo (1799), 1.506 volúmenes, sus fondos poseía las obras más representativas de la cirugía y la medicina de su época. Ambas bibliotecas se formaron con el mismo espíritu ilustrado.

Como conclusión diremos, que el funcionamiento de la Biblioteca fue regulado de forma ordenada durante todo el periodo de su existencia por distintos Estatutos, Ordenanzas y Reglamentos que hicieron que la institución se mantuviera de forma reglada a través de los distintos periodos de su existencia. Apoyada por una dotación económica cuya principal fuente de financiación fueron los profesores y alumnos, que es complementada con la que aportaba la Hacienda Real. (Remón Rodríguez 2017).

\section{FUENTES DOCUMENTALES}

- Archivo de la Universidad de Cádiz (AUCA). Libro de Cuentas del Real Colegio de Cirugía de Cádiz. 1751-1780. [libro 32]. L-44 RC.

- Archivo de la Universidad de Cádiz (AUCA). Libro de Cuentas del Real Colegio. 1754-1772. [libro 35]. L-47 RC.

- Archivo de la Universidad de Cádiz (AUCA). Libro de Cuentas del Real Colegio de Medicina y Cirugía. 1781-1795. [libro 33]. L-45 RC.

- Archivo de la Universidad de Cádiz (AUCA). Libro de Cuentas del Real Colegio de Medicina y Cirugía. 1792-1836. [libro S/N]. L-46 RC.

- Archivo de la Universidad de Cádiz (AUCA). Libro de Cuentas en el que se anota cuenta y razón de los intereses del fondo de caja del Real Colegio de Cirugía. 17671780. [libro 37]. L-53 RC. 
- Archivo de la Universidad de Cádiz (AUCA). Libro [copiador] de Reales Órdenes y decretos en favor del Real Hospital Cirugía, Real Colegio Seminario de Cádiz e individuos que lo componen: 1708-1763.Año 1749; 1751.L-54 RC.

- Archivo de la Universidad de Cádiz (AUCA). Libros de Actas de la [Junta] del Real Colegio de Cirugía y Real Colegio de Medicina y Cirugía, años: 1725-1832 [libro Tercero]. L24 RC.

- Archivo de la Universidad de Cádiz (AUCA). Libro de noticias de propuestas y deliberaciones, así como de los gastos del Real Colegio de Cirugía. Año: 1751-1814 [Libro 1]. L-22 RC.

- Archivo de la Universidad de Cádiz (AUCA). Libros de Actas de la [Junta] de la Junta del Colegio Nacional de Medicina y Cirugía, años: 1832-1836. [libro Cuarto]. L-25 RC.

- Archivo de la Universidad de Cádiz (AUCA). Libro copiador de actas de la Junta Escolástica del Colegio Nacional de Medicina y Cirugía. Años 1837-1840, [Libro quinto], 1837. L-26 RC.

- Archivo de la Universidad de Cádiz (AUCA). Libro de Actas de la [Junta] del Colegio Nacional de Medicina y Cirugía. Años 1840-1844. [Libro Sexto]. L-27 RC.

\section{REFERENCIAS BIBLIOGRÁFICAS}

- Campos Díez, M.S., 1999. El Real Tribunal del Protomedicato castellano (siglos xiv-xix). 1999. Cuenca: Universidad de Castilla-La Mancha. ISBN: 978-84-8995-8791.

- Ferrer, D. 1967a. El Real Colegio de Cirugía de Cádiz. En: AUla Militar de Cultura (ed.), Cádiz en la carrera de las Indias. Cádiz: Gobierno Militar, pp. 89 117.

- Ferrer, D. 1967b. Historia del Real Colegio de Cirugía de la Armada de Cádiz. Cádiz: Servicio de Publicaciones de la Universidad.

- Ferrer, D. 1983. Historia del Real Colegio de Cirugía de la Armada de Cádiz. Cádiz: Servicio de Publicaciones de la Universidad.

- GonzÁlez GonzÁlez, F.J. 2010. Las Bibliotecas de la Armada. En: M. GARCíA Moreno (ed.), Libros y bibliotecas: Tesoros del Ministerio de Defensa. Madrid: Ministeriod de Defensa, pp. 155-265. ISBN 97-88-4978-1605-2.

- Márquez Espinós, C. y Orozco Acuaviva, A. 1983. Estudio sobre el origen de la contribución económica de los cirujanos navales en la carrera de Indias al real 
Colegio de Cirugía de Cádiz. Primeras Jornadas de Historia de la Medicina Hispanoamericana. Cádiz: Diputación Provincial.

- Orozco Acuaviva, A. 2001. Los Reales Colegios de Cirugía en España. En: M.E. RODRÍGUEZ PÉREZ Y X. (coord.) MARTíINEZ BARBOSA (eds.), Medicina NovoHispana siglo XVIII. México: Universidad Autónoma de México, Historia General de la Medicina de México, pp. 385-392. ISBN 968-36-9801-8.

- Real Colegio de Medicina y Cirugía de CÁdiz 1791. Ordenanzas de S.M. que se deben observar en el Colegio de Medicina y Cirugía establecido en la ciudad de Cádiz: y por el cuerpo de sus profesores en la Real Armada, para gobierno del mismo Colegio, asistencia al hospital, y servicio de los buques de gue. Madrid: En la oficina de Benito Cano.

- Remón Rodríguez, A., 2017. El libro médico-científico en la Biblioteca del Real Colegio de Cirugía de Cádiz. 2017. Cádiz: Servicio de Publicaciones de la Universidad. ISBN 84-98-2864-33. 